\title{
Encoding information for future action: Memory for to-be-performed tasks versus memory for to-be-recalled tasks
}

\author{
ASHER KORIAT, HASIDA BEN-ZUR, and ALUMIT NUSSBAUM \\ University of Haifa, Haifa, Israel
}

\begin{abstract}
What is the nature of the representation underlying memory for future tasks such as calling the doctor or buying milk? If this representation consists of a verbal instruction that is translated into action at the time of retrieval, then memory should be better when tested via verbatim recall of the instruction than when tested via actual performance. Three experiments rejected this possibility, indicating better memory for a perform mode of report than for a recall mode of report. This was true in Experiment 1 in which subjects saw a series of verbal instructions (e.g., "move the eraser," "lift the cup," "touch the ashtray"), with advance information regarding the mode of report required during testing. In Experiment 2, the advance cue was valid only in $75 \%$ of the trials. Memory depended more heavily on the expected mode of report than on the actual mode of report, suggesting that the perform superiority is due to processes that occur during encoding. In Experiment 3, subjects learned 20 phrases depicting minitasks. More tasks were remembered by subjects tested via performance than by subjects tested via verbatim recall. A second part of Experiment 3 also indicated superior memory when a perform test was expected, regardless of which mode of report was actually required. The results were compared with the finding that subject-performed tasks are better remembered than are their verbal instructions, which suggests that the representation underlying memory for future assignments may take advantage of the imaginal-enactive properties of the envisaged acts. Other possible differences between memory for to-be-recalled tasks and memory for to-be-performed tasks are discussed.
\end{abstract}

In recent years, there has been a growing interest in the study of memory for action. Much of this research has centered on the memory for past activities (e.g., Anderson, 1984; Backman, Nilsson, \& Chalom, 1986; Cohen, 1981, 1983; Johnson, 1988; Kausler \& Hakami, 1983; Koriat \& Ben-Zur, 1988; Koriat, Ben-Zur, \& Sheffer, 1988). However, there are many instances in everyday life in which what we have to remember is not an act that we have already accomplished but one that has to be performed in the future. In some instances, the information pertaining to future acts must be retained for only a short interval. For example, when preparing a new dish on the basis of a written recipe, a person often has to retain a series of specific directions in short-term memory before carrying them out. A similar process occurs when one consults an operating manual in an attempt to install or operate new equipment. In other instances, memory for to-be-performed activities must extend over longer time intervals, as when a waiter must remember

The study was conducted at the Institute of Information Processing and Decision Making, University of Haifa. We are grateful to Alan Baddeley, Johannes Engelkamp, Margaret Intons-Peterson, and Norman Slamecka for their comments on an earlier version. We wish to thank Shiri Pearlman, Hamutal Pines, and Michal Sion for their help in setting up and conducting the experiments. Requests for reprints should be addressed to Asher Koriat, Department of Psychology, University of Haifa, Haifa, Israel. orders (see Bennett, 1983) or when a person plans to do a number of errands in the afternoon. In all of these examples, one is faced with the problem of remembering a series of future actions. Future actions constitute a special class of to-be-remembered events, in that they represent intended or envisaged occurrences rather than actually perceived ones. It is rather surprising that there has been little experimental work on how the content of future acts is represented in memory. In fact, it is not known whether the representation of to-be-performed assignments differs in any way from the representation of the corresponding verbal description of these assignments.

Consider, for example, a situation in which one has to remember to buy a quart of milk on the way home, call the doctor, and walk the dog. How are these future actions encoded in memory? One possibility is that the information is internally represented in an abstract propositional form, perhaps in the form of a list of verbal instructions. When the occasion for performing the act occurs, the instruction is translated into action. A second possibility is that the information is coded in a format that is more intimately tied to action, perhaps in the form of an imagined action schema. Thus, one may visualize oneself carrying out the act at the proper time and place. A third possibility is that a hybrid format is employed, in which some components (perhaps the motor components) are verbally encoded and others (perhaps the object and location components) are retained in some imaginal form. 
Recent research on memory for subject-performed tasks (SPTs; see Backman \& Nilsson, 1984; Cohen, 1981, 1983) may shed some light on the nature of the encoding of action events in general, although this research focused on the memory for past, rather than future, activities. In the SPT paradigm introduced by Cohen (1981), subjects are asked to perform several minitasks and their memory for these tasks is subsequently tested. A fairly consistent finding is that memory for SPTs is markedly superior to memory for verbal material. For example, in Cohen's study (1981, Experiment 1), immediate recall averaged $53.1 \%$ for SPTs and $44.1 \%$ for the verbal instructions. The figures for a delayed recall test were $35.6 \%$ and $24.5 \%$, respectively. Similar, and sometimes even greater, differences were reported by Backman and Nilsson (1984), Backman et al. (1986), Helstrup (1986, 1987), and others. The superior recall of SPTs has been generally attributed to their multimodal, rich properties, assumed to result in richer memorial representations than those formed for the verbal instructions alone (see Backman et al., 1986).

The SPT superiority that was demonstrated with regard to memory for past events was used in the present study to clarify the nature of encoding of future actions. SPT studies typically compare memory for past activities with memory for verbal instructions, using oral or written recall. In the present study, in contrast, subjects were always presented with verbal descriptions of minitasks, and they expected to be tested either by actually performing the tasks or by recalling the verbal descriptions themselves. If memory for future actions is mediated by verbal descriptions of these actions, we should expect inferior memory for to-be-performed tasks than for to-be-recalled tasks. This is because the translation of the verbal command into action presumably requires an additional mental operation during retrieval. On the other hand, if subjects are able to encode future tasks in a form that is more directly translatable into action, perhaps they can then form richer representations that take advantage of the multimodal, contextually rich properties of motor acts. If so, we may expect superior memory for to-be-performed tasks than for to-be-recalled tasks. Thus, in the same way that past SPTs have been found to yield better recall than do verbal instructions, we would expect superior memory for to-be-performed assignments than for to-be-recalled assignments.

An ancillary question of the present study concerned the possibility of qualitative differences between the memory for to-be-recalled and to-be-performed tasks, similar to those observed between memory for verbal material and memory for SPTs (see Cohen, 1981; Cohen \& Stewart, 1982). Such differences may indicate that information encoded for the purpose of future action is organized differently from information that is retained for future recall.

\section{EXPERIMENT 1}

In Experiment 1, the subjects were presented with a short list of verbal commands describing minitasks (e.g., "touch the stone," "lift the ashtray," "move the pencil"). In some trials, we instructed the subjects to memorize the minitasks for future performance, and we subsequently tested their memory by asking them to perform the acts (the perform condition); in other trials, we instructed them to memorize the verbal commands, and we tested their memory through verbatim recall (the recall condition).

\section{Method}

Subjects. The subjects were 19 females and 11 males. Thirteen were psychology students who participated in the experiment for course credit; the remaining subjects were volunteers from various sources. Their average age was 27.8 years (range, 18 to 40 years)

Stimulus materials. Eighty $12 \times 8 \mathrm{~cm}$ cards were used, arranged in pairs, with a "mode-of-report" card followed by an "assignment" card. Twenty of the mode-of-report cards contained the word RECALL and 20 contained the word PERFORM (in Hebrew). Each of the assignment cards included a list of three or four Hebrew sentences phrased in the singular imperative form (e.g., "lift the ashtray"), each of which appeared in a separate row. The sentences were formed by pairing one of five verb terms (operations)- touch, move, lift, tap with, and turn-with one of five noun terms ( $o b$ jects)-die, ashtray, cup, eraser, and clay. The choice of operation and object terms for each sentence was random except that each of the terms was equally represented across all assignment cards and that no operation or object term was repeated on the same card.

Procedure. The experiment was conducted individually with each subject. The subjects were told that they would be required to memorize a series of three or four sentences and that before the presentation of each series they would be informed whether their memory would be tested through verbatim recall or through action performance. They then were presented with detailed definitions of the operation terms as follows: MOVE means hold the object, move it a few centimeters away on the table, and bring it back to its original position; LIFT means hold the object, lift it a few centimeters above the table, and return it to its original place; rouch means put the fingers on the object for a couple of seconds; TURN means turn the object upside down on the table; and TAP WITH means hold the object and tap with it on the table twice. The subjects were instructed to take their hands off the object after each act. Following the definition of each term, the subjects were asked to practice the operation on a cigarette lighter that was placed on the table. The overall structure of the experiment was then explained: "A card depicting the mode-of-report cue (RECALL or PERFORM) will be followed by an assignment card containing the series of acts to be memorized. When the latter is removed, you will be required to either repeat the sentences verbatim or perform the actions described in the same order as displayed. After completing the report, you will be asked to state your confidence in the correctness of your recall."

The experiment began with four practice trials (two with a recall mode of report and two with a perform mode of report), using three objects: a box, a lighter, and a stone. The assignment cards included three sentences each and used the five operations described above. The practice objects then were replaced by five experimental objects: a playing die, an ashtray, a plastic cup, an eraser, and a piece 
of modeling clay. These were placed on the table in two rows in front of the subject, with three objects in the row closest to the subject and two in the row behind. The location of the objects was systematically varied from subject to subject but remained constant for each subject across trials. The objects remained in full view throughout the entire experiment.

The order of presentation of the 40 experimental trials was as follows: The assignment cards were kept in the same exact order for all subjects, so that the first two assignment cards included three sentences each, the next two cards included four sentences each, and so on. The mode-of-report cue (RECALL/ PERFORM) changed systematically between trials, except that for half of the subjects the RECALL cue appeared in all odd-numbered trials, whereas for the remaining half it appeared in all even-numbered trials.

The cards were presented as follows: First, the mode-of-report card was placed on the table for $1 \mathrm{sec}$, and then the assignment card was placed on top of it and remained there either for $9 \mathrm{sec}$ (three sentences) or for $12 \mathrm{sec}$ (four sentences). The two cards then were removed, and the subjects either recalled the sentences or performed the acts. At the end of each trial, the subjects estimated on a $0-100$ scale the likelihood that their response was perfectly correct.

When the experiment was completed, the subjects filled out a brief questionnaire regarding various aspects of the experiment.

\section{Results}

The first four trials of Experiment 1 were treated as warm-up trials and were not included in the analyses. Also, there were 20 trials throughout the experiment in which the wrong mode of report was used by the subject (e.g., recall instead of perform), and the results from these trials were eliminated.

Overall memory performance. Two measures of overall memory performance were derived. The first, assignment recall, was the percentage of trials for which recall was perfect, that is, all the sentences were correctly communicated in their original order. The means of the percentage of assignments recalled are presented in the first row of Table 1, by mode of report and number of sentences. A two-way analysis of variance (ANOVA) on these means indicated a highly significant effect for mode of report $[F(1,29)=19.05, p<.0001]$, with the perform mode evidencing a $15 \%$ advantage over the recall mode. Memory was better for three-sentence assignments than for four-sentence assignments $[F(1,29)=148.84$, $p<.0001]$. The effect of mode of report was somewhat stronger for four-sentence assignments than for threesentence assignments (18\% vs. $11 \%$ advantage, respectively), but this interaction was not significant $[F(1,29)$

Table 1

Means of Memory Measures as a Function of Number of Sentences and Mode of Report in Experiment 1

Mode of Report

For Three Sentences For Four Sentences

\begin{tabular}{|c|c|c|c|c|c|}
\hline \multirow[b]{2}{*}{ Row } & \multirow[b]{2}{*}{ Memory Measure } & & \multirow[b]{2}{*}{ all } & \multirow[b]{2}{*}{ Perform } \\
\hline & & Recall & Perform & & \\
\hline 1 & Assignment recall & 61.0 & 72.1 & 26.1 & 44.1 \\
\hline 2 & Act recall & 78.4 & 84.0 & 56.0 & 68.5 \\
\hline 3 & Object recall & 92.5 & 94.7 & 76.4 & 84.7 \\
\hline 4 & Operation recall & 82.4 & 87.1 & 64.9 & 74.4 \\
\hline 5 & Order-free recall & 79.2 & 85.2 & 59.7 & 72.8 \\
\hline 6 & Confidence ratings & 87.4 & 92.7 & 68.1 & 81.7 \\
\hline
\end{tabular}

$=2.49]$. One-way ANOVAs yielded significant effects of mode of report both for three-sentence assignments $[F(1,29)=6.60, p<.02]$ and for four-sentence assignments $[F(1,29)=25.32, p<.0001]$. Thus, the subjects evidenced better memory for verbally presented minitasks when they had to communicate them in action than when they had to repeat them verbatim.

A second, more refined, measure of overall memory was act recall, that is, the percentage of sentences correctly communicated in the proper ordinal position across all trials. When the subjects reported fewer sentences than were actually presented, we assumed that the $n$ sentences reported corresponded to the first $n$ sentences in the assignment card, unless indicated otherwise by the subject (e.g., "lift the die," "move the cup," "forgot the third sentence," "tap with the ashtray"). The pertinent means appear in row 2 of Table 1. A two-way ANOVA on these means yielded $F(1,29)=114.80, p<.0001$, for number of sentences, and $F(1,29)=18.08, p<.001$, for mode of report. The interaction was also significant $[F(1,29)=4.40, p<.05]$, indicating a stronger effect of mode of report for four-sentence assignments than for three-sentence assignments.

Memory for operations and memory for objects. We also analyzed the results separately for each of the two components (objects and operations) in an attempt to uncover possible qualitative differences between the recall and perform conditions. In 12 instances, the subjects provided only one of the two components of a sentence; these were not included in scoring for correct component recall. As with the previous index, the communicated component of each act was compared with the component of the act occupying the same ordinal position in the assignment card.

Rows 3 and 4 of Table 1 present the mean percentages of object recall and operation recall, respectively. A twoway ANOVA for object recall yielded $F(1,29)=41.33$, $p<.0001$, for number of sentences, $F(1,29)=16.72$, $p<.001$, for mode of report, and $F(1,29)=4.27$, $p<.05$, for the interaction. A similar ANOVA on operation recall yielded $F(1,29)=82.15, p<.0001$, for number of sentences, $F(1,29)=12.35, p<.005$, for mode of report, and $F(1,29)=2.82, p<.11$, for the interaction. Thus, the perform mode of report yielded better memory for both object and operation components, with a somewhat stronger effect for four-sentence assignments than for three-sentence assignments.

Objects were better recalled $(87.1 \%)$ than were operations $(77.2 \%)$; this difference was found equally for the perform and recall conditions. Thus, a three-way ANOVA, mode of report (recall vs. perform) $\times$ component (object vs. operation) $\times$ number of sentences (three vs. four), yielded no significant interactions. The superior memory for object terms cannot be explained by their relative position in the sentence, since in Hebrew (as in English) the verb term precedes the object term.

Item memory and order memory. Is it possible that the perform condition yields enhanced memory because it allows better retention of order information than does 
the recall condition? To examine this possibility, an orderfree memory score was calculated, consisting of the number of correct acts (operation + object) reported, irrespective of order. The means of this measure appear in row 5 of Table 1. It can be seen that even when report order is ignored, the perform condition still yields better performance than does the recall condition $[F(1,29)=17.66$, $p<.001]$.

Another question concerning order information is whether the tagging of ordinal position is attached more strongly to the object component or to the operation component. There were 83 instances across subjects and items in which all objects and all operations were correctly reported but not necessarily in their correct position. In 47 of these, more objects than operations were recalled in their correct position; in only 19 instances, the reverse pattern was found (in the remaining 17 instances, there was a tie). This pattern was equally evident in the recall and the perform conditions. Apparently, in both conditions, objects serve as better carriers of order information than do operations.

Subjective confidence. Mean confidence ratings appear in row 6 of Table 1. These were lower for foursentence assignments than for three-sentence assignments $[F(1,29)=77.50, p<.0001]$ and for the perform condition than the recall condition $[F(1,29)=43.65$, $p<.0001]$, with the difference being stronger for foursentence assignments than for three-sentence assignments $[F(1,29)=21.02, p<.0001]$.

Verbal reports. The answers to the postexperimental questionnaire also indicated that the subjects found the perform task easier than the recall task. Thus, when asked to rate the relative difficulty of the perform and recall conditions from 1 (perform condition much more difficult) to 5 (recall condition much more difficult), the mean rating was 3.83 , significantly higher than 3 (no difference) $[t(29)=4.34, p<.001]$. The subjects judged that they needed more time to memorize the sentences in the recall condition than in the perform condition $[t(29)=3.75$, $p<.001]$. They also reported that operations were more difficult to remember than were objects $[t(28)=2.59$, $p<.05]$ and that they spent more time memorizing the operations than the objects $[t(29)=3.75, p<.001]$.

The subjects were also asked to introspect about their strategies for remembering objects and operations in the recall and perform conditions. Table 2 presents the number of subjects who indicated reliance on verbal rehearsal and the number of those reporting use of imaginal encoding. Among the 16 subjects who reported having used the same learning strategy in the recall and perform conditions, there was a strong tendency to use visual imagery to encode the sequence of objects while verbally rehearsing the operation terms. The remaining 13 subjects who reported using differential strategies evidenced an additional tendency to rely more heavily on verbal rehearsal in the recall condition and on imagery in the perform condition. In fact, all 13 subjects reported relying on imagery in the encoding of objects in the perform condition and on verbal rehearsal in the encoding of operations in the
Table 2

Number of Subjects Reporting Use of Verbal Rehearsal and Imaginal Encoding, by Component Objects and Operations and Mode of Report for Experiments 1 and 2

\begin{tabular}{|c|c|c|c|c|}
\hline \multirow[b]{2}{*}{ Group } & \multicolumn{2}{|c|}{ Objects } & \multicolumn{2}{|c|}{ Operations } \\
\hline & $\begin{array}{c}\text { Verbal } \\
\text { Rehearsal }\end{array}$ & $\begin{array}{l}\text { Imaginal } \\
\text { Encoding }\end{array}$ & $\begin{array}{c}\text { Verbal } \\
\text { Rehearsal }\end{array}$ & $\begin{array}{l}\text { Imaginal } \\
\text { Encoding }\end{array}$ \\
\hline \multicolumn{5}{|c|}{ Experiment 1} \\
\hline Same Strategy & 0 & 16 & 15 & 1 \\
\hline Different Strategies & & & & \\
\hline Recall & 10 & 3 & 13 & 0 \\
\hline Perform & 0 & 13 & 5 & 8 \\
\hline \multicolumn{5}{|c|}{ Experiment 2} \\
\hline Same Strategy & 1 & 20 & 19 & 2 \\
\hline Different Strategies & & & & \\
\hline Recall & 18 & 1 & 18 & 1 \\
\hline Perform & 0 & 19 & 7 & 12 \\
\hline
\end{tabular}

recall condition. No systematic relationship was found between reported strategy and actual memory performance.

\section{Discussion}

The results of Experiment 1 clearly indicate that remembering verbal instructions for future performance is superior to remembering them for future recall. This effect was observed across a variety of memory measures and was somewhat more pronounced for four-sentence assignments than for three-sentence assignments. Also, the perform superiority was found for item information and order information, as well as for object recall and operation recall.

These results are analogous to those obtained in studies utilizing the SPT methodology (e.g., Backman \& Nilsson, 1984; Cohen, 1983). This suggests that encoding information for future performance may have much in common with the memory for tasks that have been performed by the subject in the past. The results are also consistent with findings indicating that motoric enactment of sentences enhances memory (Saltz \& Dixon, 1982). Apparently, when subjects are required to memorize verbal commands for future performance, they do not simply rehearse these commands during the retention interval and then translate them into action during testing. Rather, they may encode the commands in an imaginal form, taking advantage of the richness of the visual and motor codes that underlie action performance. Indeed, the subjects reported that they were more likely to imagine the performance of the assignments in the perform condition than in the recall condition.

We also explored the possibility that perform memory and recall memory are organized differently. However, the analyses pertaining to the retention of order information and the comparison between object and operation recall did not support this possibility.

\section{EXPERIMENT 2}

In interpreting the results of Experiment 1, we attributed the superiority of perform memory over recall 
memory to differences in the encoding operations employed in the two conditions. However, we do not know whether this superiority is indeed due to processes that occur during learning or to processes that take place during testing. This is because, in Experiment 1, the mode of report used in testing memory was always the same as that indicated in the advance cue. In Experiment 2, in contrast, the cue mode was valid in $75 \%$ of the trials, whereas, in the remaining (surprise) trials, the subjects expected one mode of report but were actually tested using the second mode of report. If encoding processes are the more critical ones, memory should be better when subjects expect to perform the assignment but are then required to recite it than when they expect verbatim recall but are later tested via actual performance.

\section{Method}

Subjects. The subjects were 40 University of Haifa psychology students ( 28 females, 12 males) who participated in the experiment for course credit. Their average age was $\mathbf{2 2 . 9}$ years (range 19 to 29 years). None had participated in Experiment 1.

Stimulus materials. There were 156 stimulus cards, arranged in triplets. The first (cue) and third (test) cards in each triplet contained either the word RECALL or the word PERFORM. All cue cards were yellow; all test cards were green. The middle (assignment) card was white and included three or four sentences, as in Experiment 1

Procedure. Five objects were used: a playing die, a plastic cup, an eraser, a lighter, and a stone. The five operations were the same as those in Experiment 1. The procedure was similar to that of Experiment 1 except for the following: We began the experiment by placing the five objects on the table in their proper spatial locations. After reading the definitions of each operation term, the subjects were asked to practice on several of the objects. Following some practice with three-sentence assignments, which they were required to learn either for verbatim recall or for performance, the subjects were told about the structure of the experiment. Thus, they were told that three cards would be presented on each trial and that the cue card would be valid in most, but not in all, trials. The subjects were encouraged to assume that the cue card was valid but were told to always use the mode of report indicated on the test card. Finally, they were given two practice trials, one with a valid cue and one with a nonvalid cue.

The experiment included 48 experimental trials. These were preceded by four warm-up trials that appeared with a valid cue. The experimental trials were ordered as follows: The assignment and test cards were in the same order for all subjects. This order was random except that half of the three-sentence assignments and half of the four-sentence assignments were followed by a PERFORM test card and the other half by a RECALL test card. The validity manipulation was produced by changing the cue cards across two equal groups of subjects. For each group, each block of 12 successive trials included nine valid cues and three nonvalid cues; however, if a trial contained a nonvalid cue for one group of subjects, it was always used with a valid cue for the other group. Each subject's 12 surprise trials included 3 three-sentence assignments and 3 foursentence assignments in the recall-perform pattern and the rest in the perform-recall pattern.

The cue and test cards were exposed for 2 sec each; the assignment card appeared for $9 \mathrm{sec}$ (three sentences) or $12 \mathrm{sec}$ (four sentences). A brief questionnaire was administered at the end of the experiment.

\section{Results}

The same memory measures as those in Experiment 1 were calculated for each cue mode $\times$ test mode combination; the means are presented in Table 3 . The means for each of the valid cue cells (i.e., recall-recall and perform-perform) were based on 18 trials per subject; those for each of the nonvalid cue cells were based on six trials per subject.

A cue mode $\times$ test mode ANOVA on the percentage of assignments recalled yielded $F(1,39)=15.37$, $p<.0005$, for cue mode, $F(1,39)=2.07$, n.s., for test mode, and $F(1,39)=11.62, p<.005$, for the interaction. Thus, encoding mode affected performance, whereas test mode did not. The interaction derives primarily from the fact that recall was better in the valid cue condition than in the nonvalid cue condition $[t(39)=3.41$, $p<.001]$. The effect of cue validity, however, was significant only for the recall mode of encoding [ $t(39)=$ $3.54, p<.001$ ], whereas performance was equally high under both testing conditions for the perform mode of encoding $[t(39)=0.71$, n.s.]. This suggests that when information is encoded for future performance, memory remains high even when a verbal recall test is used.

When only the valid cue conditions were considered, the perform mode of report evidenced only a $4 \%$ advantage over the recall mode of report $[F(1,39)=2.01$, $p<.17$, as opposed to a $15 \%$ advantage in Experiment 1 . This difference may be due to the lesser reliability of the report cue in Experiment 2.

The same general pattern of results was found for act recall. A cue mode $\times$ test mode ANOVA yielded $F(1,39)$ $=12.05, p<.005$, for cue mode, $F<1$, for test mode, and $F(1,39)=18.36, p<.0001$, for the interaction. The valid cue conditions indicated a nearly significant advantage for the perform mode $[F(1,39)=3.52, p<.07]$. Among the nonvalid cue conditions, the perform-recall condition evidenced better recall than the recall-perform condition $[F(1,39)=5.91, p<.02]$.

The results for the remaining four measures listed in Table 3 yielded very similar effects to those reported for assignment and act recall. Thus, for all four measures, two-way ANOVAs indicated significant effects only for

Table 3

Means of Memory Measures for Different Combinations of Cue and Test Conditions in Experiment 2

\begin{tabular}{clccccc}
\hline & & \multicolumn{4}{c}{ Test Condition } \\
\cline { 3 - 4 } \cline { 6 - 7 } Row & Memory Measure & Recall & Perform & & Recall & Perform \\
\cline { 3 - 4 } \cline { 6 - 7 } 1 & Assignment recall & 49.8 & 38.8 & & 51.3 & 54.0 \\
2 & Act recall & 68.5 & 61.5 & & 68.3 & 71.9 \\
3 & Object recall & 86.0 & 82.9 & & 87.7 & 88.5 \\
4 & Operation recall & 74.7 & 68.0 & & 71.9 & 76.7 \\
5 & Order-free recall & 71.8 & 66.2 & & 72.4 & 74.7 \\
6 & Confidence ratings & 82.4 & 80.3 & & 83.0 & 84.7 \\
\hline
\end{tabular}


the cue mode and none for the test mode. The interaction was significant for operation recall $(p<.0005)$ and order-free recall $(p<.005)$; the interaction was near significant for object recall $(p<.06)$ and confidence ratings $(p<.07)$. The means (see Table 3 ) indicate superior performance with a perform cue than with a recall cue. Also, within each cue type, performance was consistently better when the test mode was the same as the cue mode than when it differed from it.

All analyses reported above were repeated, using number of sentences as an additional factor in the ANOVA. This factor yielded highly significant main effects for all memory measures listed in Table 3 , but it yielded no significant interactions with the other factors.

Finally, we should note several additional aspects of the data. First, as in Experiment 1, object recall was superior to operation recall; this was true for each of the cue mode $x$ test mode combinations (see Table 3 ). This superiority was stronger for the two nonvalid cue conditions (a 15\% advantage) than for the two valid cue conditions (a $12 \%$ advantage). Thus, a two-way ANOVA, cue validity (valid vs. nonvalid cue) $\times$ component (object vs. operation), yielded $F(1,39)=107.60, p<.0001$, for component, $F(1,39)=15.58, p<.001$, for valid ity, and $F(1,39)=7.94, p<.01$, for the interaction. The interaction suggests that when subjects must use a mode of report different from that anticipated during encoding, memory for operations is more strongly impaired than memory for objects.

Second, as may be expected, the means for order-free recall are generally higher than those for act recall (which requires order-specific recall). The difference, however, was somewhat larger for the nonvalid cue than for the valid cue conditions. Thus, a two-way cue validity $x$ order requirement (act recall vs. order-free recall) ANOVA yielded $F(1,39)=15.71, p<.001$, for validity, $F(1,39)=25.47, p<.0001$, for order requirement, and $F(1,39)=4.55, p<.05$, for the interaction. The interaction suggests that the use of an unexpected mode of testing results in greater damage to order information than to item information.

In the postexperimental questionnaire, 21 subjects reported using the same encoding strategy regardless of the cue presented and 19 subjects reported differential en coding strategies for recall and perform trials. The dis tribution of subjects according to the encoding strategy used for objects and operations appears in Table 2, and, as may be seen, it is remarkably similar to that obtained in Experiment 1. We should note that, as far as actual memory performance is concerned, both groups yielded very similar results, indicating a significant effect for mode of encoding. However, the interaction between mode of encoding and mode of testing was somewhat stronger for the group reporting differential strategies for recall and perform trials.

\section{Discussion}

The aim of Experiment 2 was to determine the locus of the perform advantage observed in Experiment 1. The results clearly indicated that memory performance depends more heavily on the type of cue presented during learning than on the mode of report used in testing memory. For all six memory measures, mode of encoding exerted a significant main effect, with perform cues resulting in better performance than recall cues. In contrast, mode of testing had no overall systematic effect on performance.

Over and above the main effect of cue mode, the use of an unexpected mode of report during testing seemed to impair memory performance. Thus, within each expected mode of report, performance was superior when the test mode accorded with the expected mode than when it differed from it. This suggests that subjects can benefit from advance knowledge regarding mode of testing, apparently through the use of differential encoding strategies. It should be noted, however, that the impairment in memory resulting from the use of an unexpected test mode was greater when a recall test was expected than when a perform test was expected. It would seem that there is some advantage to encoding verbal instructions in a manner that stresses their future enactment, even when the actual test requires verbatim recitation of the instructions.

Finally, when only the valid cue conditions are considered, the results partly replicate those of Experiment 1, indicating an advantage for the perform condition over the recall condition. Although this advantage was consistently obtained across all memory measures, it was less marked than in Experiment 1 and not significant. Perhaps, because of the unreliability of the advance cue in Experiment 2 , the subjects were more reluctant to vary their encoding strategies in accordance with the requirements of the anticipated mode of testing (although this is not supported by the subjects' verbal reports; see Table 2). Nevertheless, the interactions observed between cue mode and test mode, as well as the overall effect of cue mode, suggest that the subjects did rely to some extent on the advance cues provided.

Altogether, the results of Experiment 2 point to differences in encoding as the main source of the perform advantage observed in Experiment 1. Apparently, when expecting to enact verbal instructions, subjects retain the information in a medium that takes advantage of the sensorimotor features of the acts. This conclusion is similar to that of Saltz and Dixon (1982) on the effects of motoric enactment. In their study, enactment during learning enhanced cued recall of the sentences whether or not subjects were required to enact the sentence during the test phase. Thus, perhaps the effects of encoding mode observed in the present study are due to the perform cue's activating a symbolic (mental) enactment of the to-beperformed tasks.

\section{EXPERIMENT 3}

The experimental paradigm used in Experiments 1 and 2 differs in important respects from some of the typical everyday situations in which one has to remember to per- 
form several acts in the future, such as doing a list of errands. Most notably, in the latter situation, the assignments are drawn from a relatively broad repertoire of possible activities and remembering depends more heavily on long-term retention than on short-term retention. The experimental paradigm of Experiments 1 and 2, in contrast, required the use of a miniature grammar with a restricted set of verb and noun terms. This was necessary to facilitate comparisons between the recall and perform modes of report. Furthermore, the memory task used in Experiments 1 and 2 apparently taps short-term, rather than long-term, memory.

In Experiment 3, we attempted to extend the conclusions of Experiments 1 and 2 to a situation requiring longterm memory for a larger and more varied series of minitasks. The procedure closely paralleled that typically employed in SPT research (e.g., Cohen, 1983), except that we experimentally manipulated the nature of the response (i.e., mode of report) rather than the nature of the stimulus event. Thus, in Part 1 of Experiment 3, the subjects were presented with 20 sentences, each depicting a minitask of the sort used in SPT research. In the recall condition, the subjects were instructed to learn the minitasks for future recall, whereas, in the perform condition, they were instructed to learn the tasks for future performance. The list was presented for four learning-testing trials. In the test phase, the subjects were instructed to either recall or perform the tasks (according to the mode expected) in any order they wished.

In Part 2 of Experiment 3, the subjects learned a new list of tasks, each group expecting the same mode of report as that used in Part 1. However, only half of the subjects in each group were tested using that mode of report; the other half were tested using the other, unexpected, mode of report. Finally, a second learning-testing trial took place, in which the expected and actual modes of report were those used in testing recall on the preceding trial.

We expect the results of Part 1 to yield the same pattern of perform superiority as that observed in Experiments 1 and 2. If this superiority derives, as we have argued, from processes that take place during encoding, then performance in Part 2 should depend on the expected mode of report rather than on the mode of report actually used for testing memory.

\section{Method}

Subjects. The subjects were 40 University of Haifa psychology students ( 27 females, 13 males) who participated in the experiment for course credit. Their average age was 22.3 years (range 18 to 30 years).

Materials. Two lists were prepared; each list was made up of 20 Hebrew phrases each of which described a minitask. The lists were compiled from various sources (e.g., Cohen, 1981). All phrases were in the infinitive form and contained up to four words each (e.g., to smile, to stand up, to smell a flower, to knock on the door). Each phrase was printed on a separate card. Four versions of each list were prepared, differing only in the order of the sentences. The order was random, with the exception that none of the sentences appeared more than once across the first two and the last two positions of each list. Three additional lists of four sentences each were used for practice.

Design and Procedure. In Part 1 of Experiment 3, the subjects were presented with one list of sentences for four learning-testing trials; in Part 2, they were presented with the second list for two learning-testing trials. Twenty subjects received Part 1 with perform instructions; the remaining 20 subjects received Part 1 with recall instructions. On the first trial of Part 2, all subjects received the same learning instructions as they had received in Part 1. However, for the testing procedure, the subjects were further divided into two groups such that 10 subjects in each group were tested with the recall mode of report and the remaining 10 subjects were tested with the perform mode. The encoding and testing modes for the second trial of Part 2 were the same as the testing mode used on the first trial. The assignment of the two lists to Parts 1 and 2 of the experiment was counterbalanced across the four groups of subjects. A different version of the list was used on each trial; the assignment of versions to trials was also counterbalanced across subjects.

The experiment was conducted individually with each subject. It was introduced as a study of the memory for future assignments of the sort involved in everyday life (e.g., when one has to remember to call the doctor, pay a bill, etc.). The subjects in the perform condition were told that they would be presented with a series of assignments to memorize for future enactment and that, when presentation was completed, they would be required to enact all sentences one after the other in any order they wished. They were instructed that when the assignment required an object (e.g., "to cut bread"), they should enact it on (or with) an imaginary object. The subjects were then given practice with three lists of four phrases each. Each list was presented by exposing the cards one after the other; when presentation was completed, the subjects were required to enact the phrases regardless of order. They were asked to wait until the experimenter said "yes" before they carried out the next assignment. It was stressed that all phrases must be clearly communicated so that the experimenter could interpret and record each enacted phrase.

Each card was exposed for $5 \mathrm{sec}$, with about 1 -sec intervals between cards. When presentation was completed, the experimenter said, "Now perform all the assignments you remember. Order is immaterial. Wait until I say 'yes' before you proceed to the next assignment. Begin now." This instruction was partly intended to reduce recency effects. A 2 -min period was allowed for recall. This exact procedure was repeated for three more learning-testing trials.

The procedure for the recall condition was the same except that the subjects were instructed to memorize the phrases for future recall and testing required oral recitation of the phrases.

In Part 2 of Experiment 3, the subjects were told that the same exact procedure would be conducted but with a second list of assignments. For the perform-perform and the recall-recall groups, the procedure for the two trials of Part 2 was exactly the same as in Part 1. For the other two groups, the procedure changed after the first presentation of the list. That is, the perform-recall group received the same (perform) learning instructions as in Part 1, but, when presentation of the list was completed, the subjects were told that "we are now interested in your ability to remember the verbal phrases themselves," and, thus, they were required to repeat aloud all the assignments that they could remember from the list. The procedure for the second trial of Experiment 3 was similar except that the subjects were warned in advance that a verbatim recall would be required. In the recall-perform group, on the other hand, the subjects were given recall instructions during the learning phase but were then told to enact the phrases in the test phase, invoking an imaginary object when needed. In the instructions for the second trial, the subjects were warned that the phrases would have to be enacted during testing.

All responses were recorded by the experimenter. There were several instances in the perform condition when the communicated 
assignment was not clear to the experimenter (typically an extralist intrusion), and the subject was asked to spell out verbally what the assignment was. When the experiment was completed, the subjects filled out a brief questionnaire.

\section{Results}

The recall protocols were scored by classifying each of the responses into the following categories (note that, in parentheses, we have given the mean number of items per trial across the four trials of Part 1): correct act recall (14.65); partial recall, that is, correct report of operation or object $(0.31)$; extralist intrusion $(0.28)$; and repetition of a previously reported act (0.98). A lenient scoring procedure was used in scoring the protocols from the recall condition, so that inaccurate responses (e.g. , "drink water') were scored as correct if their motor enactment could not be distinguished from that of the correct response (e.g., "drink coffee")

We shall first examine the results of Part 1. Figure 1 (left panel) presents mean percentage of acts recalled for the recall and perform modes as a function of trial. For each of the trials, the perform mode yielded better memory than did the recall mode, but the effect decreased with trial. A two-way ANOVA on these results yielded $F(1,38)=7.50, p<.01$, for mode of report, $F(3,114)$ $=204.76, p<.0001$, for trial, and $F(3,114)=2.89$, $p<.05$, for the interaction. Separate one-way ANOVAs for each trial indicated a significant (or near significant) effect of mode of report for Trial $1[F(1,38)=10.90$, $p<.005]$, Trial $2[F(1,38)=5.83, p<.05]$, and Trial $3[F(1,38)=3.85, p<.06]$, but not for Trial 4

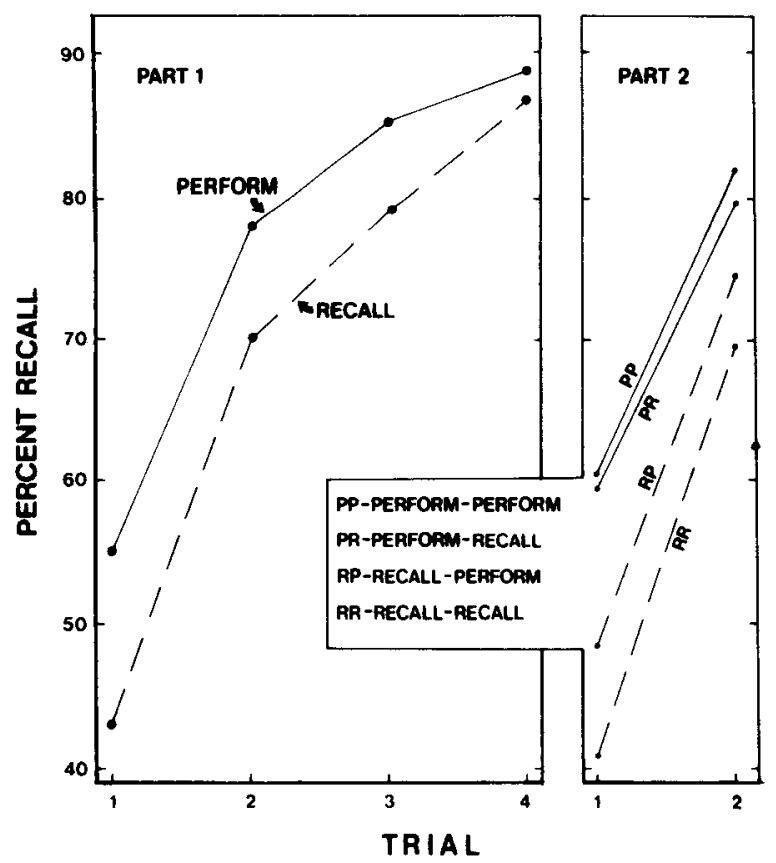

Figure 1. Mean percentages of acts recalled in Part 1 (left panel) and Part 2 (right panel) of Experiment 3 for subjects in different encoding and testing conditions.
$(F<1)$. Thus, the assignments were better remembered when they were learned for future performance than when they were learned for future recall.

We shall turn next to the results of Part 2. Mean percentage of correct recall is depicted in the right panel of Figure 1 for the four groups of subjects representing all combinations of mode of report used in Part 1 of Experiment 3 and mode of testing used in Part 2. Note that on the first trial of Part 2, the subjects always expected the same mode of report as that used in Part 1-this mode will therefore be referred to as the encoding mode. A twoway ANOVA yielded $F(1,36)=13.75, p<.001$, for encoding mode, $F(1,36)=1.07$, n.s., for test mode, and $F<1$, for the interaction. These results suggest that the perform advantage obtained in Part 1 of the experiment is most probably due to the conditions of encoding rather than to the conditions of testing. Although the perform test mode yielded slightly better memory scores than did the recall test mode, the difference was not significant.

The results of Trial 2 were very similar to those of Trial 1. A two-way ANOVA yielded $F(1,36)=4.77$, $p<.05$, for encoding mode (on Trial 1 ), and $F<1$, for test mode as well as for the interaction. Thus, the advantage of the perform subjects over the recall subjects in Part 1 carried over to Trial 2 of Part 2, despite the fact that half of the subjects in each group were required to switch to the other mode of report. Presumably, practice with the perform mode of report leads to the adoption of effective encoding strategies that are beneficial even when verbatim recall is required.

Several additional analyses were carried out in search for possible qualitative differences between memory for to-be-performed tasks and memory for to-be-recalled tasks. First, in the context of SPT research, Cohen (1981) reported flatter serial position curves for immediate memory of SPTs than for immediate memory of verbal instructions. We examined the possibility of a similar difference between the recall and perform conditions of Part 1 of Experiment 3. The results did not reveal such differences. Thus, when the data for Trial 1 were grouped into five intervals (four positions per interval), a two-way condition $\times$ interval ANOVA yielded $F(1,38)=10.90$, $p<.005$, for condition, $F(4,152)=3.45, p<.01$, for interval, and $F<1$, for the interaction. Mean percent recall for the five successive intervals was $66.2,48.7$, $55.0,51.2$, and 53.7 , for the perform condition, and 53.7, $36.2,38.7,35.0$, and 52.5 , for the recall condition. If primacy effects reflect the operation of active encoding operations, it would seem that memory for the purpose of future performance is not subject to a lesser degree of strategic control than is memory for the purpose of future recall.

Second, the subjects were more likely to repeat a previously communicated task in the recall mode than in the perform mode. Mean number of repetitions (per trial) in Part 1 was 1.31 , for the recall mode, and 0.64 for the perform mode $[F(1,38)=12.67, p<.005]$. The results of Trial 1 of Part 2 suggest that this effect is due to the 
mode of testing rather than to the mode of encoding: A two-way ANOVA on number of repetitions yielded $F(1,36)=12.42, p<.005$, for mode of testing, and $F(1,36)=2.64$, n.s., for mode of encoding. Number of repetitions for the recall and perform tests averaged 0.83 and 0.18 , respectively. Apparently, output monitoring is more effective for motor enactment than for verbal recall.

Finally, the results for individual items suggest that the effect of mode of report in Part 1 was strongest for minitasks that implied an object not present in the room (e.g., flower). There were nine such minitasks in one list and eight in the other. They yielded a perform advantage of $11 \%$, on the average, whereas the remaining minitasks, which either required an object that was present in the room (e.g., table) or did not require an object, yielded a perform advantage of only $4 \%$.

\section{Discussion}

The task used in Experiment 3 differs in important respects from that used in Experiments 1 and 2. Notably, it required the long-term retention of an unstructured list of minitasks, and memory was tested using a free (rather than a serial) format of report. Nevertheless, the results replicated the main findings from Experiments 1 and 2. First, there was a clear superiority of the perform condition over the recall condition in Part 1. The perform advantage amounted to about $12 \%$ on the first trial, similar to what was reported by Cohen $(11 \%)$ for the advantage of memory for SPTs over memory for verbal instructions (Cohen, 1981, Experiment 1).

Second, Part 2 confirmed the hypothesis that the perform superiority is due to the encoding, rather than the retrieval, phase of the task. Learning material for subsequent performance yielded better memory than did learning for subsequent recall, and this was the case regardless of the actual mode of report used.

\section{GENERAL DISCUSSION}

\section{Overview of Hypotheses and Findings}

In the present study, we have focused on a neglected issue in memory research: the encoding of actions for future performance. We contrasted two hypotheses: (1) that future tasks are encoded in a propositional form, perhaps in the form of a verbal command, and (2) that the encoding of future tasks may utilize an imaginal, sensorimotor medium that affords a relatively direct translation into action-if such is the case, the encoding of tasks for future performance may take advantage of the richness of the sensory and motor properties of the planned action to enhance retention.

Our three experiments differed in important procedural respects. Experiments 1 and 2 employed a restricted set of operations and objects and tapped short-term retention, whereas Experiment 3 used a larger and more varied set of minitasks and tested mainly for long-term memory. Nevertheless, their results were consistent in indicating two major trends. First, all three experiments yielded bet- ter memory in the perform condition than in the recall condition. This was true in Experiments 1 and 2 in which mode of report was manipulated within subjects, as well as in Experiment 3 (Part 1) in which a between-subjects design was employed.

Second, the results of Experiment 2 and of Part 2 of Experiment 3 place the locus of the perform advantage at the encoding phase rather than at the retrieval phase. In Experiment 2, memory performance was found to depend more heavily on the type of cue presented during learning than on the mode of report used in testing memory. A similar effect was observed in Part 2 of Experiment 3: Subjects expecting a perform mode of report evidenced better memory, irrespective of which mode of report was actually used during testing. In both experiments, mode of testing, as such, had little overall effect.

These results have two general implications: the first concerns the strategic control over encoding operations and the second concerns the nature of the representation underlying memory for to-be-performed tasks.

\section{Encoding Strategies Vary with Expected Mode of Report}

The results on the whole suggest that subjects employ different, probably more efficient encoding strategies when they expect task performance than when they expect verbal recall. Memory performance in Experiment 3 (Part 2) depended entirely on the expected mode of report, whereas the results of Experiment 2 yielded an additional effect for the congruency between the expected and the actual mode of report, with recall being consistently better when encoding and testing modes were the same than when they differed. This effect implies that subjects can benefit from advance knowledge regarding mode of testing (see Tversky, 1969).

Two additional observations also suggest that memory for future activities is subject to strategic control. First, the subjects' introspective reports clearly indicate reliance on encoding strategies in the perform condition that were different from those in the recall condition. Second, in Experiment 3, the perform condition yielded a serialposition curve with a clear primacy effect, similar to that found for the recall condition. Primacy effects have been generally seen as indicative of active attempts to memorize (see Helstrup, 1987).

When these results are compared with those obtained for SPT memory, it would seem that the memory for future actions is subject to a greater strategic control than is the memory for past activities. With regard to the latter, previous research suggests that memory for SPTs is largely automatic, that is, not subject to strategic encoding operations (Cohen, 1981; Cohen \& Stewart, 1982). Our results indicate that this conclusion may not apply to memory for future activities. Perhaps, the task of remembering future actions provides an organizational scheme that is not normally available in memory for SPTs, and this scheme induces a greater reliance on active encoding strategies. Indeed, Helstrup (1987) has shown that 
SPT recall also yields primacy effects when subjects are provided with some organizational scheme (e.g., to think back to the last time they performed the act or to think ahead to the next time they intend to perform such acts).

\section{The Nature of the Representation Underlying Memory for Future Actions}

The second implication concerns the nature of the encoding of future tasks. The perform superiority may be explained in terms of the dual-coding hypothesis (Paivio, 1971,1986 ), assuming that subjects rely more heavily on a propositional-verbal coding when expecting a recall test and on imaginal coding when expecting task performance. This proposal is supported by the subjects' introspective reports, which indicated greater reliance on imaginal encoding in the perform condition and on verbal rehearsal in the recall condition. It is also consistent with previous findings regarding the beneficial effect of motoric enactment on memory (see Helstrup, 1987). This effect has been attributed to the multimodal and contextually rich properties of SPTs, which promote the use of visual and motor encoding (see Backman \& Nilsson, 1984). A similar memory advantage has been observed even when the tasks were performed symbolically, in a make-believe fashion, rather than concretely (e.g., Engelkamp \& Zimmer, 1985; Helstrup, 1986; Saltz \& Dixon, 1982). Furthermore, similar to what was found in the present study, the effect of symbolic enactment appears to be due to a better encoding of the information during learning rather than to its mode of retrieval (Saltz \& Dixon, 1982).

These parallels between the memory for future tasks and the memory for concretely or symbolically performed tasks suggest that the two types of tasks share the same underlying representational code. This appears to be true despite the possibility, noted above, that the memory for future tasks affords greater strategic control than does the memory for previously performed tasks. Perhaps the encoding of future tasks entails an internal, symbolic enactment of the tasks, which enhances memory. Thus, in the same way that memory for to-be-recalled verbal material is enhanced by verbal rehearsal, memory for to-beperformed tasks might also be enhanced by a symbolic enactment of the task. In both cases, the rehearsal process consists of a repeated internal simulation of the procedure that the subject expects to carry out at the time of test, that is, either verbal recitation of the sentence or performance of the task. The differences in the expected mode of testing may, thus, guide the encoding processes activated and, consequently, the nature of the representation that is retained.

If this mode-of-representation account is accepted, two questions emerge. First, what is the nature of the nonverbal, or imaginal, code used with regard to to-beperformed tasks? In the context of memory for previously performed tasks, the question has been raised whether the effects of enactment on memory are due to the activation of visual images (Levin, 1976) or to the activation of motoric images (Saltz \& Donnenwerth-Nolan, 1981). This question is important in view of the recent evidence supporting the existence of a motor representation, distinct from visual imagery (see Engelkamp \& Zimmer, 1984; Klatzky, Pellegrino, McCloskey, \& Doherty, 1989; Saltz \& Donnenwerth-Nolan, 1981; Zimmer \& Engelkamp, 1985). We may similarly inquire whether to-be-performed tasks are encoded in a visual form or in a motor form. Our results do not permit definitive conclusions, though we should note that the introspective reports indicate a greater mention of visual imagery than of motor imagery in encoding future tasks.

The second question concerns the extent to which different codes may collaborate in the representation of future tasks. It has been proposed that SPTs are better remembered because they allow representation in terms of a number of codes--perhaps verbal, visual, and motor (see Zimmer \& Engelkamp, 1989). The same may be true for memory for future tasks. In addition, however, the verbal reports suggest the possibility that memory for future tasks is enhanced not only by reliance on a multiplicity of codes but also by the assignment of different aspects of the task to different systems of representation. Thus, in both Experiments 1 and 2, the subjects reported differential encoding strategies with regard to the object and operation components of the tasks, with objects more often retained via an imaginal scheme and with operations memorized through verbal rehearsal. Such "split encoding" may enhance memory either by increasing the distinctiveness of the individual elements (see Mohr, Engelkamp, \& Zimmer, 1989) or by making better use of the memory-storage system.

Although the mode-of-representation account of the perform advantage is consistent with several observations from both the present study and previous work on pastperformed tasks, other accounts are also tenable. One is that a perform mode of encoding induces the extraction of relational aspects and enhances interactive, unitary coding (see Marschark, Richman, Yuille, \& Hunt, 1987). Another is that to-be-performed tasks are more likely to induce encoding with respect to the self than are to-berecalled tasks (see Greenwald, 1981).

\section{The Relation of the Present Work to Prospective Memory}

The present work may have some bearing on the phenomenon of prospective remembering, that is, the memory to perform certain acts in the future (e.g., to show up for an appointment or return a book to the library; see Winograd, 1988). Prospective memory research, however, has focused almost entirely on the cue aspect (i.e., on remembering the intention to perform something in the future or on remembering to probe one's memory for that intention at the appropriate time) and neglected the issue of how the content of what has to be carried out is remembered. This neglect apparently derives from the assumption, common among researchers of prospective memory (e.g., Harris, 1984; Wilkins \& Baddeley, 1978), that in everyday life the content component (e.g., turn 
off the oven) is often trivially easy to remember, and the main difficulty lies in activating the cue component, that is, in retrieving the content under the proper circumstances (e.g., turning off the oven when the cake is ready). Consistent with this emphasis on the cue aspect, the tasks typically employed in the study of prospective remembering have been too simple (e.g., mailing a letter or pressing a button; see Harris, 1984) to afford insights into the representation and retention of the content information. However, it is clear that the content component is implicated in all prospective memory episodes and that it becomes particularly critical when one has to plan several activities for future performance. We are familiar with everyday situations in which we know that we were supposed to do something but cannot remember what it was. Such instances reflect a failure to retrieve the content component.

The present study focused on memory for the informational content, and it may have some bearing on the manner in which the content component of prospective remembering is encoded in memory. To study this component, at the exclusion of the cue component, we used to-beperformed tasks that make greater demands on memory than do tasks typically employed in studies of prospective remembering and we tested memory under conditions that do not rely on subjects' spontaneous probing of their memories. In retrospect, however, it is of some interest to examine whether our results also have some bearing on questions pertaining to the cue component. It is possible that an imaginal format of representation, one that takes advantage of the modality-specific, rich properties of a future task, should be beneficial not only for the memory of the content of the task but also for the subsequent cuing of this memory under the proper conditions. If a future task (e.g., buy newspaper) is encoded in terms of sensorimotor properties of the task that allow a relatively direct translation into action, perhaps this type of encoding is also more likely to facilitate the activation of memory by the appropriate external circumstances (e.g., the view of a newspaper stand) than is a verbally based encoding.

\section{REFERENCES}

ANDERson, R. E. (1984). Did I do it or did I only imagine doing it? Journal of Experimental Psychology: General, 113, 594-613.

BACKMAN, L., \& Nilsson, L.-G., (1984). Aging effects in free recall: An exception to the rule. Human Learning, 3, 53-69.

Backman, L., Nilsson, L.-G., \& Chalom, D. (1986). New evidence on the nature of the encoding of action events. Memory \& Cognition, 14, 339-346.

BenNeTt, H. L. (1983). Remembering drink orders: The memory skills of cocktail waitresses. Human Learning, 2, 157-169.

CoHen, R. L. (1981). On the generality of some memory laws. Scandinavian Journal of Psychology, 22, 267-281.

CoHEN, R. L. (1983). The effect of encoding variables on the free recall of words and action events. Memory \& Cognition, 11, 575-582.

COHEN, R. L., \& STEWART, M. (1982). How to avoid developmental effects in free recall. Scandinavian Journal of Psychology, 23, 9-15.
EngelKamp, J., \& Zimmer, H. D. (1984). Motor programme information as a separable memory unit. Psychological Research, 46, 283-299.

EngelKamp, J., \& Zimmer, H. D. (1985). Motor programs and their relation to semantic memory. German Journal of Psychology, 9 , 239-254.

Greenwald, A. G. (1981). Self and memory. In G. H. Bower (Ed.), The psychology of learning and motivation (Vol. 15, pp. 201-236). New York: Academic Press.

HaRris, J. E. (1984). Remembering to do things: A forgotten topic. In J. E. Harris \& P. E. Morris (Eds.), Everyday memory actions and absent mindedness (pp. 71-92). London: Academic Press.

Helstrup, T. (1986). Separate memory laws for recall of performed acts? Scandinavian Journal of Psychology, 27, 1-29.

Helstrup, T. (1987). One, two, or three memories? A problem-solving approach to memory for performed acts. Acta Psychologica, 66, 37-68.

JoHNSON, M. K. (1988). Reality monitoring: An experimental phenomenological approach. Journal of Experimental Psychology: General, 117, 390-394.

KaUSLER, D. H., \& HAKAmi, M. K. (1983). Memory for activities: Adult age differences and intentionality. Developmental Psychology, 19, 889-894.

Klatzky, R. L., Pellegrino, J. W., McCloskey, B. P., \& DoHERTY, S. (1989). Can you squeeze a tomato? The role of motor representation in semantic sensibility judgments. Journal of Memory \& Language, 28, 56-77.

Koriat, A., \& Ben-Zur, H. (1988). Remembering that I did it: Processes and deficits in output monitoring. In $\mathbf{M}$. Gruneberg, P. Morris, \& R. Sykes (Eds.), Practical aspects of memory: Current research and issues (Vol. 1, pp. 203-208). Chichester: Wiley.

Koriat, A., Ben-Zur, H., \& Sheffer, D. (1988). Telling the same story twice: Output monitoring and age. Journal of Memory \& Language, 27, 23-39.

LEVIN, J. R. (1976). What have we learned about maximizing what children learn? In G. R. Levin \& V. L. Allen (Eds.), Cognitive learning in children (pp. 105-134). New York: Academic Press.

Marschark, M., Richman, C. L., Yuille, J. C., \& Hunt, R. R. (1987). The role of imagery in memory: On shared and distinctive information. Psychological Bulletin, 102, 28-41.

Mohr, G., Engelkamp, J., \& Zimmer, H. D. (1989). Recall and recognition of self-performed acts. Psychological Research, 51, 181-187.

PaIvio, A. (1971). Imagery and verbal processes. New York: Holt, Rinehart \& Winston.

Paivio, A. (1986). Mental representations: A dual-coding approach. Oxford: Oxford University Press.

SALTZ, E., \& Dixon, D. (1982). Let's pretend: The role of motoric imagery in memory for sentences and words. Joumal of Experimental Child Psychology, 34, 77-92.

SALTZ, E., \& DonNenWerth-Nolan, S. (1981). Does motoric imagery facilitate memory for sentences? A selective interference test. Journal of Verbal Learning \& Verbal Behavior, 20, 322-332.

TVersky, B. (1969). Pictorial and verbal encoding in a short-term memory task. Perception \& Psychophysics, 6, 225-233.

Wilkins, A. J., \& Baddeley, A. D. (1978). Remembering to recall in everyday life: An approach to absent-mindedness. In M. Gruneberg, P. Morris, \& R. Sykes (Eds.), Practical aspects of memory. London: Academic Press.

WinOGRAD, E. (1988). Some observations on prospective remembering. In M. Gruneberg, P. Morris, \& R. Sykes (Eds.), Practical aspects of memory: Current research and issues (Vol. 1, pp. 348-353). Chichester: Wiley.

Zimmer, H. D., \& Engelkamp, J. (1985). An attempt to distinguish between kinematic and motor memory components. Acta Psychologica, 58, 81-106.

Zimmer, H. D., \& ENGElkamp, J. (1989). One, two or three memories: Some comments and new findings. Acta Psychologica, 70, 293-304.

(Manuscript received November 3, 1989; revision accepted for publication February 23, 1990.) 